\title{
UM MÉTODO PRIMAL-DUAL DE PONTOS INTERIORES E EXTERIORES BARREIRA LOGARÍTMICA MODIFICADA COM ESTRATÉGIAS DE EXTRAPOLAÇÃO CÚBICA E CONVERGÊNCIA GLOBAL.
}

\author{
R. B. N. Pinheiro ${ }^{1}$, A. R. Balbo ${ }^{2}$.
}

${ }^{1}$ Programa de Pós-Graduação em Engenharia Elétrica, Faculdade de Engenharia de Bauru FEB/UNESP, Bauru-SP (ribenopi@ hotmail.com).

${ }^{2}$ Departamento de Matemática, Faculdade de Ciências - FC/UNESP, Bauru-SP.

Resumo. Neste trabalho apresentamos um método previsor-corretor primal-dual de pontos interiores e exteriores barreira logarítmica modificada com estratégias de extrapolação cúbica e convergência global (MPIBLMCG-EX). Na definição do algoritmo proposto, a função barreira logarítmica modificada existe e auxilia o método em sua inicialização com pontos inviáveis que pertencem à região de inviabilidade relaxada (ampliada). Porém, a inviabilidade pode ocorrer em pontos que não estão próximos à fronteira relaxada ou não pertençam a esta região, consequentemente, implicando na não existência da função barreira logarítmica modificada. Para suprir essa dificuldade uma extrapolação cúbica, que preserva as diferenciais de primeira e segunda ordem nas proximidades da fronteira, é aplicada ao método; no procedimento previsor, são realizadas atualizações do parâmetro de barreira nos resíduos das restrições de complementaridade, considerando aproximações de $1^{a}$. ordem do sistema de direções de busca, enquanto que no procedimento corretor, incluímos os termos quadráticos não-lineares dos resíduos citados, que foram desprezados no procedimento previsor. Consideramos também a estratégia de convergência global para o método, a qual utiliza uma variante do método de Levenberg-Marquardt para atualizar a matriz dual normal da função lagrangiana caso esta não seja definida positiva. Neste caso, esta matriz é redefinida para restrições primais, de igualdade, desigualdade e variáveis canalizadas, incorporando variáveis duais e matrizes diagonais relativas às restrições de complementaridade. Uma implementação deste método, realizada em Matlab 6.1, mostrou-se eficiente quando aplicada em problemas de FPO, da área de Sistema Elétrico de Potência (SEP) em Engenharia Elétrica, cuja função objetivo e o conjunto de restrições são funções não-lineares e não-convexas. Neste trabalho apresentamos os resultados da aplicação do método em destaque para o sistema elétrico IEEE118.

Palavras-chave: Método de ponto interior, extrapolação cúbica, convergência global. 


\section{O MÉTODO PREVISOR-CORRETOR PRIMAL-DUAL DE PONTOS INTERIORES BARREIRA LOGARÍTMICA MODIFICADA (MPIBLM).}

Para a definição do método previsor-corretor primal-dual de pontos interiores barreira logarítmica modificada (MPIBLM), consideramos o seguinte modelo geral de otimização nãolinear com restrições de igualdade, de desigualdades canalizadas e de variáveis canalizadas:

$$
\left\{\begin{array}{ll}
\text { Min } & f(\mathbf{x}) \\
\text { s.a: } & \\
& \mathbf{g}(\mathbf{x})=\mathbf{0} \\
& \mathbf{u}_{\mathbf{1}} \leq \mathbf{h}(\mathbf{x}) \leq \mathbf{u}_{\mathbf{2}} \\
& \mathbf{l}_{\mathbf{1}} \leq \mathbf{x} \leq \mathbf{l}_{\mathbf{2}}
\end{array} ;\right.
$$

em que: $\mathbf{x} \in \mathbb{R}^{n}, f(\mathbf{x}): \mathbb{R}^{n} \rightarrow \mathbb{R}$ é a função objetivo, $\mathbf{g}(\mathbf{x}): \mathbb{R}^{n} \rightarrow \mathbb{R}^{m}$ e $\mathbf{h}(\mathbf{x}): \mathbb{R}^{n} \rightarrow \mathbb{R}^{r}$ são funções de classe $C_{2}$ e a condição de qualificação de restrições é a aquela proposta por (Mangasarian, et al., 1976). O problema (1.1) é transformado em problema de otimização nãolinear irrestrito e equivalente, de acordo com [1] a qual utiliza a função barreira modificada apresentada por [2], a seguir:

$$
\left\{\begin{array}{c}
\text { Min } \quad L(\boldsymbol{\omega})=f(\mathbf{x})-\mu \sum_{i=1}^{r}\left(\delta_{1}\right)_{i} \ln \left(z_{1}^{\diamond}\right)_{i}-\mu \sum_{i=1}^{r}\left(\delta_{2}\right)_{i} \ln \left(z_{2}^{\diamond}\right)_{i}- \\
-\mu \sum_{j=1}^{n}\left(\delta_{3}\right)_{j} \ln \left(z_{3}^{\diamond}\right)_{j}-\mu \sum_{j=1}^{n}\left(\delta_{4}\right)_{j} \ln \left(z_{4}^{\diamond}\right)_{j}+\sum_{t=1}^{m}\left(\lambda_{0}\right)_{t} g_{t}(\mathbf{x})+ \\
+\sum_{i=1}^{r}\left[\left(\lambda_{1}\right)_{i}\left(-h_{i}(\mathbf{x})+\left(u_{1}\right)_{i}+\left(z_{1}\right)_{i}\right)+\left(\lambda_{2}\right)_{i}\left(h_{i}(\mathbf{x})-\left(u_{2}\right)_{i}+\left(z_{2}\right)_{i}\right)\right]+ \\
+\sum_{j=1}^{n}\left[\left(\lambda_{3}\right)_{j}\left(-x_{j}+\left(l_{1}\right)_{j}+\left(z_{3}\right)_{j}\right)+\left(\lambda_{4}\right)_{j}\left(x_{j}-\left(l_{2}\right)_{j}+\left(z_{4}\right)_{j}\right)\right]
\end{array} ;\right.
$$

onde: $\mu \in \mathbb{R}_{*}^{+}$é o parâmetro de barreira; $\boldsymbol{\delta}_{\mathbf{1}}, \boldsymbol{\delta}_{\mathbf{2}} \in \mathbb{R}_{+}^{r}$ e $\boldsymbol{\delta}_{\mathbf{3}}, \boldsymbol{\delta}_{\mathbf{4}} \in \mathbb{R}_{+}^{n}$ são vetores estimadores dos multiplicadores de Lagrange; $\lambda_{\mathbf{0}} \in \mathbb{R}_{*}^{m}$ é o vetor de multiplicador de Lagrange referente às restrições de igualdade; $\lambda_{1}, \lambda_{2} \in \mathbb{R}_{+}^{r}$ são os vetores de multiplicadores de Lagrange referente às restrições funcionais canalizadas; $\lambda_{3}, \lambda_{4} \in \mathbb{R}_{+}^{n}$ são os vetores de multiplicadores de lagrange referente às variáveis canalizadas; $\mathbf{z}_{\mathbf{1}}, \mathbf{z}_{\mathbf{2}} \in \mathbb{R}^{r}$ e $\quad \mathbf{z}_{\mathbf{3}}, \mathbf{z}_{\mathbf{4}} \in \mathbb{R}^{n}$ são variáveis de folga; $\left(z_{1}^{\diamond}\right)_{i}=\left(1+\frac{z_{1}}{\mu}\right)_{i},\left(z_{2}^{\diamond}\right)_{i}=\left(1+\frac{z_{2}}{\mu}\right)_{i},\left(z_{3}^{\diamond}\right)_{j}=\left(1+\frac{z_{3}}{\mu}\right)_{j}$ e $\left(z_{4}^{\diamond}\right)_{j}=\left(1+\frac{z_{4}}{\mu}\right)_{j}$ são as variáveis primais definidas pela relaxação das restrições de desigualdade de (1.1) em relação às variáveis de folga $\mathbf{z}_{1}>\mathbf{0}, \mathbf{z}_{2}>\mathbf{0}, \mathbf{z}_{3}>\mathbf{0}$ e $\mathbf{z}_{4}>\mathbf{0}$ tais que: $\mathbf{z}_{1}>-\boldsymbol{\mu}, \mathbf{z}_{2}>-\boldsymbol{\mu}, \mathbf{z}_{3}>-\boldsymbol{\mu}$ e $\mathbf{z}_{4}>-\boldsymbol{\mu}$; 
$\boldsymbol{\omega}=\left(\mathbf{x}, \mathbf{z}_{\mathbf{i}}, \lambda_{\mathbf{0}}, \boldsymbol{\lambda}_{\mathbf{i}}\right)^{T} \in \mathbb{R}_{*}^{m+5 n+4 r}$ é o vetor de variáveis da função lagrangiana aumentada interior $L(\boldsymbol{\omega})$. As condições de KKT são recuperadas ao aplicarmos a condição necessária de otimalidade $\nabla L(\boldsymbol{\omega})=\mathbf{0}$. Essas condições são apresentadas a seguir:

$$
\nabla L(\boldsymbol{\omega})=\left\{\begin{array}{lcl}
\nabla_{\mathbf{x}} L= & \nabla f(\mathbf{x})+\nabla \mathbf{g}(\mathbf{x})^{\mathbf{T}} \boldsymbol{\lambda}_{\mathbf{0}}+\nabla \mathbf{h}(\mathbf{x})^{\mathbf{T}}\left(\boldsymbol{\lambda}_{2}-\lambda_{1}\right)-\lambda_{3}+\lambda_{4}=\mathbf{0} \\
\nabla_{\lambda_{0}} L= & \mathbf{g}(\mathbf{x}) & =\mathbf{0} \\
\nabla_{\lambda_{1}} L= & -\mathbf{h}(\mathbf{x})+\mathbf{u}_{1}+\mathbf{z}_{1} & =\mathbf{0} \\
\nabla_{\lambda_{2}} L= & \mathbf{h}(\mathbf{x})-\mathbf{u}_{2}+\mathbf{z}_{2} & =\mathbf{0} \\
\nabla_{\lambda_{3}} L= & -\mathbf{x}+\mathbf{l}_{1}+\mathbf{z}_{3} & =\mathbf{0} \\
\nabla_{\lambda_{4}} L= & \mathbf{x}-\mathbf{l}_{2}+\mathbf{z}_{4} & =\mathbf{0} \\
\nabla_{\mathbf{z}_{1}} L= & \bar{Z}_{1} \lambda_{1}-\mu \boldsymbol{\delta}_{1} & =\mathbf{0} \\
\nabla_{\mathbf{z}_{2}} L= & \bar{Z}_{2} \lambda_{2}-\mu \boldsymbol{\delta}_{2} & =\mathbf{0} \\
\nabla_{\mathbf{z}_{3}} L= & \bar{Z}_{3} \lambda_{3}-\mu \boldsymbol{\delta}_{3} & =\mathbf{0} \\
\nabla_{\mathbf{z}_{4}} L= & \bar{Z}_{4} \lambda_{4}-\mu \boldsymbol{\delta}_{4} & \mathbf{0}
\end{array} ;\right.
$$

onde: $\nabla \mathbf{g}(\mathbf{x}) \in \mathbb{R}^{m \times n}$ e $\nabla \mathbf{h}(\mathbf{x}) \in \mathbb{R}^{r \times n}$ são, respectivamente, as matrizes jacobianas dos funcionais $\mathbf{g}(\mathbf{x})$ e $\mathbf{h}(\mathbf{x})$ e $\bar{Z}_{i}^{-1}=\operatorname{diag}\left(\left(\mu+z_{i}\right)^{-1}\right), i=1, \ldots, 4$. Em cada equação do sistema nãolinear $\nabla L(\boldsymbol{\omega})=\mathbf{0}$ linearizaremos por um aproximante de Taylor de primeira ordem. Assim, temos o sistema linear a seguir:

$$
A_{k} \mathbf{d}_{\mathbf{\omega}}^{\mathbf{k}}=\mathbf{b}^{\mathbf{k}}
$$

onde: 


$$
A_{k}=\left(\begin{array}{cccccccccc}
\mathbf{K} & 0 & 0 & 0 & 0 & \nabla \mathbf{g}\left(\mathbf{x}^{\mathbf{k}}\right)^{T} & -\nabla \mathbf{h}\left(\mathbf{x}^{\mathbf{k}}\right)^{T} & \nabla \mathbf{h}\left(\mathbf{x}^{\mathbf{k}}\right)^{T} & -I_{n} & I_{n} \\
\nabla \mathbf{g}\left(\mathbf{x}^{\mathbf{k}}\right) & 0 & 0 & 0 & 0 & 0 & 0 & 0 & 0 & 0 \\
-\nabla \mathbf{h}\left(\mathbf{x}^{\mathbf{k}}\right) & I_{r} & 0 & 0 & 0 & 0 & 0 & 0 & 0 & 0 \\
\nabla \mathbf{h}\left(\mathbf{x}^{\mathbf{k}}\right) & 0 & I_{r} & 0 & 0 & 0 & 0 & 0 & 0 & 0 \\
-I_{n} & 0 & 0 & I_{n} & 0 & 0 & 0 & 0 & 0 & 0 \\
I_{n} & 0 & 0 & 0 & I_{n} & 0 & 0 & 0 & 0 & 0 \\
0 & \Lambda_{1_{k}} & 0 & 0 & 0 & 0 & \bar{Z}_{1_{k}} & 0 & 0 & 0 \\
0 & 0 & \Lambda_{2_{k}} & 0 & 0 & 0 & 0 & \bar{Z}_{2_{k}} & 0 & 0 \\
0 & 0 & 0 & \Lambda_{3_{k}} & 0 & 0 & 0 & 0 & \bar{Z}_{3_{k}} & 0 \\
0 & 0 & 0 & 0 & \Lambda_{4_{k}} & 0 & 0 & 0 & 0 & \bar{Z}_{4_{k}}
\end{array}\right) ;(1.5)
$$

em que: $\quad \mathrm{K}=\nabla^{2} f\left(\mathbf{x}^{\mathbf{k}}\right)+\sum_{t=1}^{m}\left[\left(\lambda_{0}^{k}\right)_{t} \nabla^{2} g_{t}\left(\mathbf{x}^{\mathbf{k}}\right)\right]+\sum_{i=1}^{r}\left[\left(\lambda_{2}^{k}-\lambda_{1}^{k}\right)_{i} \nabla^{2} h_{i}\left(\mathbf{x}^{\mathbf{k}}\right)\right] ; \quad I_{n} \quad$ é a matriz identidade de ordem $n ; I_{r}$ é a matriz identidade de ordem $r$ e $\Lambda_{i_{k}}=\operatorname{diag}\left(\lambda_{i}^{k}\right)$; $\mathbf{d}_{\boldsymbol{\omega}}^{\mathbf{k}}=\left(\mathbf{d}_{\mathbf{x}}^{\mathbf{k}}, \mathbf{d}_{\mathbf{z}_{\mathbf{i}}}^{\mathbf{k}}, \mathbf{d}_{\lambda_{\mathbf{0}}}^{\mathbf{k}}, \mathbf{d}_{\lambda_{\mathbf{i}}}^{\mathbf{k}}\right)^{\mathbf{T}}, i=1, \ldots, 4$ e $\mathbf{b}^{\mathbf{k}}$ é o vetor residual definido por:

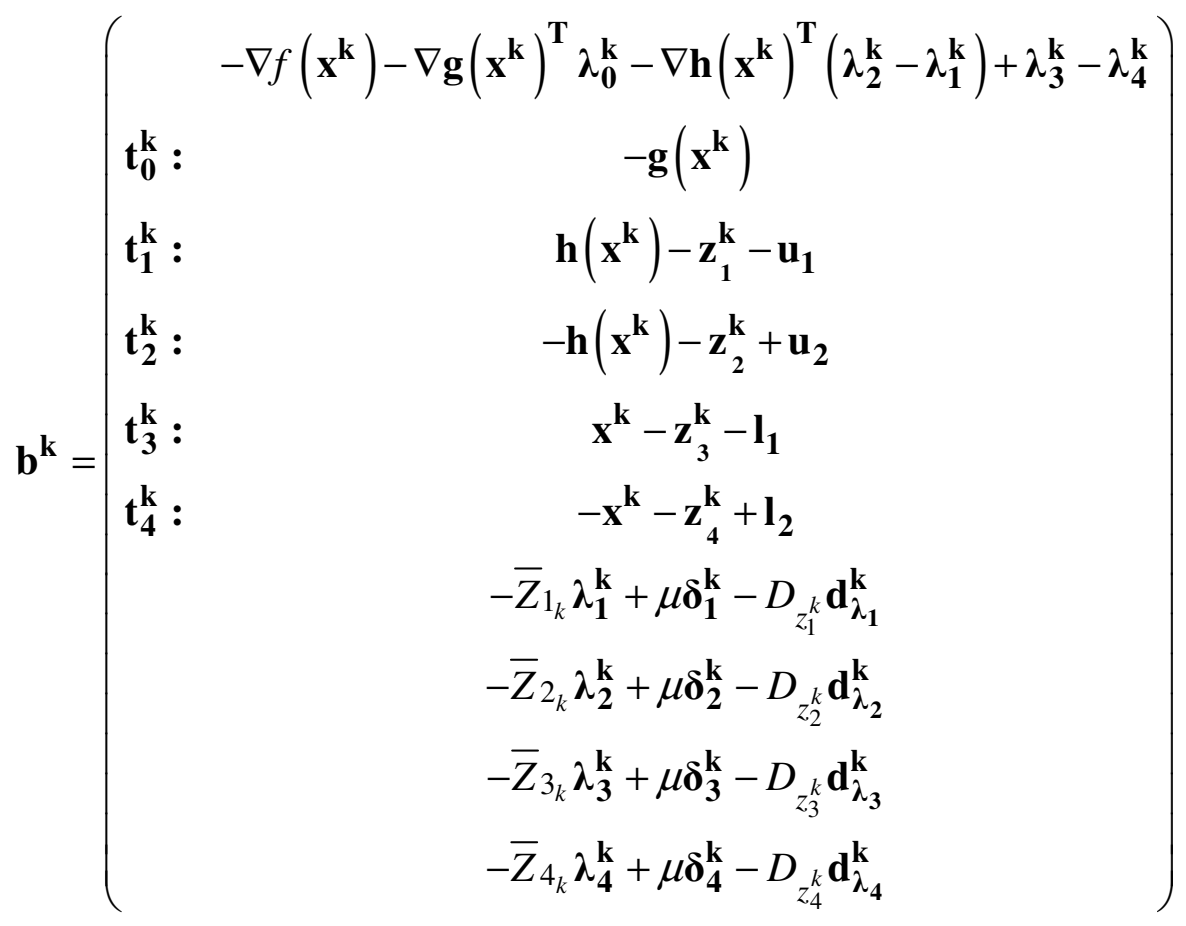


em que: $D_{z_{i}^{k}}=\operatorname{diag}\left(d_{z_{i}}^{k}\right), i=1, \ldots, 4$. Para a definição do procedimento previsor, desprezamos os resíduos não-lineares $D_{z_{i}^{\mathbf{k}}} \mathbf{d}_{\lambda_{\mathbf{i}}}^{\mathbf{k}}, i=1, \ldots, 4$, isto é, a priori definimos esses resíduos como nulos, pois desconhecemos essas direções. Essa estratégia é uma variante do método previsor-corretor de [3], diferenciando-se do procedimento definido por esses autores, pois consideramos o parâmetro de barreira, definido nas condições de complementaridade apresentadas em (1.3), no procedimento previsor, para o cálculo das direções de busca. Assim, com procedimentos algébricos, definimos, a seguir, o quadro de direções primais e duais:

$$
\begin{aligned}
& \mathbf{d}_{\lambda_{\mathbf{0}}}^{\mathbf{k}}=-\left(\nabla \mathbf{g}\left(\mathbf{x}^{\mathbf{k}}\right) \theta_{k}^{-1} \nabla \mathbf{g}\left(\mathbf{x}^{\mathbf{k}}\right)^{\mathbf{T}}\right)^{-1}\left(\nabla \mathbf{g}\left(\mathbf{x}^{\mathbf{k}}\right) \theta_{k}^{-1}\left(\nabla f\left(\mathbf{x}^{\mathbf{k}}\right)+\mathbf{c}^{\mathbf{k}}+\mu \varphi^{\mathbf{k}}\right)+\mathbf{t}_{\mathbf{0}}^{\mathbf{k}}\right)-\lambda_{\mathbf{0}}^{\mathbf{k}} ; \\
& \mathbf{d}_{\mathbf{x}}^{\mathbf{k}}=\quad-\theta_{k}^{-1}\left(\nabla f\left(\mathbf{x}^{\mathbf{k}}\right)+\mathbf{c}^{\mathbf{k}}+\mu \varphi^{\mathbf{k}}+\nabla \mathbf{g}\left(\mathbf{x}^{\mathbf{k}}\right)^{\mathbf{T}} \lambda_{0}^{\mathbf{k}}+\nabla \mathbf{g}\left(\mathbf{x}^{\mathbf{k}}\right)^{\mathbf{T}} \mathbf{d}_{\lambda_{0}}^{\mathbf{k}}\right) \\
& \mathbf{d}_{\mathbf{z}_{1}}^{\mathbf{k}}=\quad \nabla \mathbf{h}\left(\mathbf{x}^{\mathbf{k}}\right) \mathbf{d}_{\mathbf{x}}^{\mathbf{k}}+\mathbf{t}_{\mathbf{1}}^{\mathbf{k}} ; \\
& \mathbf{d}_{\mathbf{z}_{2}}^{\mathbf{k}}=\quad-\nabla \mathbf{h}\left(\mathbf{x}^{\mathbf{k}}\right) \mathbf{d}_{\mathbf{x}}^{\mathbf{k}}+\mathbf{t}_{2}^{\mathbf{k}} \\
& \mathbf{d}_{\mathbf{z}_{3}}^{\mathrm{k}}=\quad \mathbf{d}_{\mathbf{x}}^{\mathrm{k}}+\mathbf{t}_{3}^{\mathrm{k}} \\
& \mathbf{d}_{\mathbf{z}_{4}}^{\mathbf{k}}=-\quad-\mathbf{d}_{\mathbf{x}}^{\mathbf{k}}+\mathbf{t}_{\mathbf{4}}^{\mathbf{k}} ; \\
& \mathbf{d}_{\lambda_{1}}^{\mathbf{k}}=\quad-\bar{Z}_{1_{k}}^{-1} \Lambda_{1_{k}} \mathbf{d}_{\mathbf{z}_{\mathbf{1}}}^{\mathbf{k}}+\mu \bar{Z}_{1_{k}}^{-1} \boldsymbol{\delta}_{\mathbf{1}}^{\mathbf{k}}-\lambda_{\mathbf{1}}^{\mathbf{k}} ; \\
& \mathbf{d}_{\lambda_{2}}^{\mathbf{k}}=\quad-\bar{Z}_{2_{k}}^{-1} \Lambda_{2_{k}} \mathbf{d}_{\mathbf{z}_{2}}^{\mathbf{k}}+\mu \bar{Z}_{2_{k}}^{-1} \boldsymbol{\delta}_{2}^{\mathbf{k}}-\lambda_{2}^{\mathbf{k}} ; \\
& \mathbf{d}_{\lambda_{3}}^{\mathbf{k}}=\quad-\bar{Z}_{3_{k}}^{-1} \Lambda_{3_{k}} \mathbf{d}_{\mathbf{z}_{3}}^{\mathbf{k}}+\mu \bar{Z}_{3_{k}}^{-1} \boldsymbol{\delta}_{\mathbf{3}}^{\mathbf{k}}-\lambda_{3}^{\mathbf{k}} ; \\
& \mathbf{d}_{\lambda_{4}}^{\mathbf{k}}=\quad-\bar{Z}_{4_{k}}^{-1} \Lambda_{4_{k}} \mathbf{d}_{\mathbf{z}_{\mathbf{4}}}^{\mathbf{k}}+\mu \bar{Z}_{4_{k}}^{-1} \boldsymbol{\delta}_{\mathbf{4}}^{\mathbf{k}}-\lambda_{\mathbf{4}}^{\mathbf{k}} \text {. }
\end{aligned}
$$

onde:

$$
\begin{gathered}
\theta_{k} \in \mathbb{R}^{n \times n}: \mathrm{K}+\nabla \mathbf{h}\left(\mathbf{x}^{\mathbf{k}}\right)^{\mathbf{T}}\left(\bar{Z}_{1_{k}}^{-1} \Lambda_{1_{k}}+\bar{Z}_{2_{k}}^{-1} \Lambda_{2_{k}}\right) \nabla \mathbf{h}\left(\mathbf{x}^{\mathbf{k}}\right)+\bar{Z}_{3_{k}}^{-1} \Lambda_{3_{k}}+\bar{Z}_{4_{k}}^{-1} \Lambda_{4_{k}} \\
\mathbf{c}^{\mathbf{k}} \in \mathbb{R}^{n}: \nabla \mathbf{h}\left(\mathbf{x}^{\mathbf{k}}\right)^{\mathbf{T}}\left(\bar{Z}_{1_{k}}^{-1} \Lambda_{1_{k}} \mathbf{t}_{\mathbf{1}}^{\mathbf{k}}-\bar{Z}_{2_{k}}^{-1} \Lambda_{2_{k}} \mathbf{t}_{\mathbf{2}}^{\mathbf{k}}\right)+\bar{Z}_{3_{k}}^{-1} \Lambda_{3_{k}} \mathbf{t}_{\mathbf{3}}^{\mathbf{k}}-\bar{Z}_{4_{k}}^{-1} \Lambda_{4_{k}} \mathbf{t}_{\mathbf{4}}^{\mathbf{k}} \\
\boldsymbol{\varphi}^{\mathbf{k}} \in \mathbb{R}^{n}: \nabla \mathbf{h}\left(\mathbf{x}^{\mathbf{k}}\right)^{\mathbf{T}}\left(-\bar{Z}_{1_{k}}^{-1} \boldsymbol{\delta}_{\mathbf{1}}^{\mathbf{k}}+\bar{Z}_{2_{k}}^{-1} \boldsymbol{\delta}_{\mathbf{2}}^{\mathbf{k}}\right)-\bar{Z}_{3_{k}}^{-1} \boldsymbol{\delta}_{\mathbf{3}}^{\mathbf{k}}+\bar{Z}_{4_{k}}^{-1} \boldsymbol{\delta}_{\mathbf{4}}^{\mathbf{k}}
\end{gathered}
$$

Com as direções do procedimento previsor calculadas, inicia-se o procedimento corretor considerando os resíduos não-lineares $D_{z_{i}^{k}} \mathbf{d}_{\lambda_{\mathbf{i}}}^{\mathbf{k}}, i=1, \ldots, 4$, pois agora conhecemos essas direções. O quadro de direções do procedimento corretor é análogo ao do procedimento corretor. A diferença está na complementação de expressões algébricas sobre o vetor $\mathbf{c}^{\mathbf{k}}$, em que, para distingui-lo do procedimento previsor, o definiremos por $\tilde{\mathbf{c}} \mathbf{k}$ apresentado a seguir: 


$$
\begin{aligned}
\tilde{\mathbf{c}}^{\mathbf{k}}=\nabla \mathbf{h}\left(\mathbf{x}^{\mathbf{k}}\right)^{\mathbf{T}}\left(\bar{Z}_{1_{k}}^{-1} \Lambda_{1_{k}} \mathbf{t}_{1}^{\mathbf{k}}+\bar{Z}_{1_{k}}^{-1} D_{z_{1}} \mathbf{d}_{\lambda_{1}}^{\mathbf{k}}-\bar{Z}_{2_{k}}^{-1} \Lambda_{2_{k}} \mathbf{t}_{2}^{\mathbf{k}}-\bar{Z}_{2_{k}}^{-1} D_{z_{2}^{k}} \mathbf{d}_{\lambda_{2}}^{\mathbf{k}}\right)+ \\
+\bar{Z}_{3_{k}}^{-1} \Lambda_{3_{k}} \mathbf{t}_{3}^{\mathbf{k}}+\bar{Z}_{3_{k}}^{-1} D_{z_{3}^{k}} \mathbf{d}_{\lambda_{3}}^{\mathbf{k}}-\bar{Z}_{4_{k}}^{-1} \Lambda_{4_{k}} \mathbf{t}_{4}^{\mathbf{k}}-\bar{Z}_{4_{k}}^{-1} D_{z_{4}^{k}} \mathbf{d}_{\lambda_{4}}^{\mathbf{k}} .
\end{aligned}
$$

Com as direções corrigidas $\tilde{\mathbf{d}}_{\boldsymbol{\omega}}^{\mathbf{k}}=\left(\tilde{\mathbf{d}}_{\mathbf{x}}^{\mathbf{k}}, \tilde{\mathbf{d}}_{\mathbf{z}_{\mathbf{i}}}^{\mathbf{k}}, \tilde{\mathbf{d}}_{\lambda_{\mathbf{0}}}^{\mathbf{k}}, \tilde{\mathbf{d}}_{\lambda_{\mathbf{i}}}^{\mathbf{k}}\right)^{\mathbf{T}}, i=1, \ldots, 4$ calculadas, definimos o cálculo do tamanho do passo primal $\overline{\alpha_{p}}$ e do passo dual $\overline{\alpha_{d}}$ pela regra apresentada em [4]. Esta é apresentada a seguir:

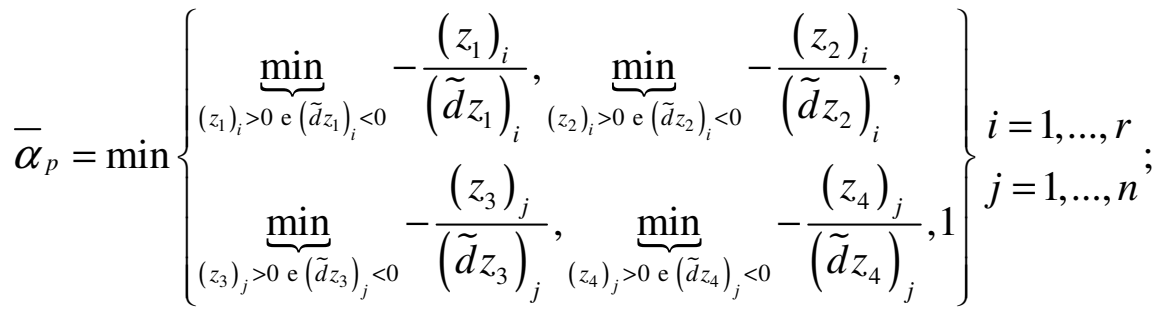

$$
\begin{aligned}
& \bar{\alpha}_{d}=\min \left\{\begin{array}{c}
\underbrace{\min }_{\left(\lambda_{i}>0 \mathrm{e}\left(d \lambda_{1}\right)_{i}<0\right.}-\frac{\left(\lambda_{1}\right)_{i}}{\left(\tilde{d} \lambda_{1}\right)_{i}}, \underbrace{\min }_{\left(\lambda_{2}\right)_{i}>0 \mathrm{e}\left(\tilde{d} \lambda_{2}\right)_{i}<0}-\frac{\left(\lambda_{2}\right)_{i}}{\left(\tilde{d} \lambda_{2}\right)_{i}}, \\
\underbrace{\min }_{\left(\lambda_{3}\right)_{j}>0 \mathrm{e}\left(\tilde{d} \lambda_{3}\right)_{j}<0}-\frac{\left(\lambda_{3}\right)_{j}}{\left(\tilde{d} \lambda_{3}\right)_{j}}, \underbrace{\min }_{\left(\lambda_{4}\right)_{j}>0 \mathrm{e}\left(\tilde{d} \lambda_{4}\right)_{j}<0}-\frac{\left(\lambda_{4}\right)_{j}}{\left(\tilde{d} \lambda_{4}\right)_{j}}, 1
\end{array}\right\} j=1, \ldots, r ., .
\end{aligned}
$$

Então, a expressão que define $\boldsymbol{\omega}^{\mathbf{k}+1}$ é definida por:

$$
\omega^{\mathbf{k}+1}=\omega^{\mathbf{k}}+\bar{\alpha} \tilde{\mathbf{d}}_{\omega}^{\mathbf{k}} .
$$

Dado um escalar $\varepsilon>0$ suficientemente pequeno, verificamos se o critério de parada do MPIBLM é satisfeito se a seguinte condição está satisfeita: $\left\|\nabla L\left(\omega^{\mathbf{k}+1}\right)\right\|_{\infty} \leq \mathcal{E}$. Caso o critério não seja satisfeito, atualizamos o parâmetro de barreira $\mu^{k}$ por uma heurística definida por:

$$
\mu^{k+1}=\alpha_{\mu} \mu^{k}
$$

em que $\alpha_{\mu} \in(0,1) \subset \mathbb{R}$ é definido previamente; atualizamos os estimadores dos multiplicadores de Lagrange $\delta_{\mathbf{i}}^{\mathbf{k}}$ pela seguinte regra:

$$
\boldsymbol{\delta}_{\mathbf{i}}^{\mathbf{k}+1}=\lambda_{\mathbf{i}}^{\mathbf{k}+1}, i=1, \ldots, 4
$$

e reiniciamos o procedimento previsor-corretor. 


\section{A ESTRATÉGIA DE CONVERGÊNCIA GLOBAL.}

Uma vez que $\theta_{k}=\frac{\partial L\left(\omega^{\mathbf{k}}\right)}{\partial \mathbf{x} \partial \mathbf{x}}$ é simétrica e definida positiva, então a Decomposição de Cholesky (DC) é possível de ser realizada. Caso a DC não seja possível uma estratégia, que pode ser encontrada em [5] em [6], é aplicada ao método. Essa estratégia é uma perturbação sobre a matriz $\theta_{k}$ a qual é definida da seguinte maneira:

$$
\hat{\theta}_{k}=\theta_{k}+\beta I_{n}
$$

onde $\beta \in \mathbb{R}_{+}$é denominado por parâmetro de damping ou parâmetro de amortecimento do método de Levenberg-Marquardt, o qual foi desenvolvido para a resolução de problemas de quadrados mínimos. Assim, enquanto a DC não for satisfeita, a perturbação (1.17) é aplicada, isto é, essa estratégia transforma o MPIBLM de tal forma a determinar e garantir uma sequência de direções de descida para a determinação de novos pontos, convertendo-o em um método primal-dual com estratégia de convergência global (CG), isto é, o MPIBLM transforma-se em MPIBLMCG. O parâmetro $\beta$ influencia tanto na direção quando no tamanho do passo, assim, o procedimento de Levenberg-Marquardt não necessita de uma busca linear para descobrir o tamanho do passo a ser dado em cada direção de busca em uma iteração qualquer. Seja $\operatorname{lm}$ o número de iterações necessárias para tornar a matriz $\theta_{k}$ em definida positiva, então temos o processo iterativo definido a seguir:

$$
\beta^{l m+1}=\beta^{l m}\left(\frac{\mu_{1}+\sqrt{(\sqrt{5}-1)^{2} \mu^{2}+\mu_{1}^{2}}}{2 \mu_{1}}\right)
$$

em que $\mu_{1}=\mu^{k-1}$ e $\beta^{k}$ é definido por $\beta^{k}=\beta^{l m+1}$. Para uma iteração que passa do procedimento corretor para o procedimento previsor, ou seja, para uma iteração $k+1$, podemos atualizar o valor de $\beta$ de acordo com as definições a seguir, que propõe uma modificação na definição do parâmetro de amortecimento em relação àquela encontrada em [5]:

i) Se $L\left(\boldsymbol{\omega}^{\mathbf{k}}\right)-L\left(\boldsymbol{\omega}^{\mathbf{k}+\mathbf{1}}\right)<0,25$, então $\beta$ é atualizado pela heurística:

$$
\beta^{k+1}=\frac{\beta^{k}}{3}
$$

ii) Se $L\left(\boldsymbol{\omega}^{\mathbf{k}}\right)-L\left(\boldsymbol{\omega}^{\mathbf{k}+\mathbf{1}}\right)>0,25$, então $\beta$ é atualizado pela heurística (1.18);

iii) Se $0,25 \leq L\left(\boldsymbol{\omega}^{\mathbf{k}}\right)-L\left(\boldsymbol{\omega}^{\mathbf{k}+\mathbf{1}}\right) \leq 0,75$, então $\beta$ não será alterado. 


\section{A ESTRATÉGIA DE EXTRAPOLAÇÃO CÚBICA.}

Na definição do algoritmo proposto, a penalização logarítmica modificada existe e auxilia o MPIBLMCG em seu procedimento com pontos inviáveis que pertencem à região de viabilidade relaxada (ampliada ou aumentada). Porém, a inviabilidade pode ocorrer em pontos que estão próximos ou que não pertençam à região relaxada, consequente, implicando na nãoexistência da função barreira logarítmica modificada. Para suprir essa dificuldade, uma extrapolação cúbica, que preserva as diferenciais de primeira e de segunda ordem, é aplicada ao MPIBLMCG. Essa extrapolação é uma variante do ajuste quadrático para funções barreiras modificadas que pode ser encontrado em [7] e, com mais detalhes, em [8]. Por simplicidade, definiremos por $z$ uma componente qualquer dos vetores $\mathbf{z}_{1}, \mathbf{z}_{2}, \mathbf{z}_{3}$ ou $\mathbf{z}_{4}$.

Seja $\tau \in(0,1) \subset \mathbb{R}:$ o parâmetro de proximidade da variável de folga $z$ à fronteira interior relaxada. Se $z \geq-\tau \mu$, então a função barreira modificada $\Psi(z)$ é definida por: $\Psi(z)=\ln \left(1+\frac{z}{\mu}\right)$. Caso contrário, se $z<-\tau \mu$, uma extrapolação cúbica é definida pelo polinômio a seguir:

$$
\Psi(z)=\frac{q_{3}}{6} z^{3}+\frac{q_{2}}{2} z^{2}+q_{1} z+q_{0}
$$

em que:

$$
\left\{\begin{array}{lll}
q_{0} & = & \ln (1-\tau)+\frac{11 \tau^{3}-15 \tau^{2}+6 \tau}{6(1-\tau)^{3}} \\
q_{1} & = & \frac{\left(3 \tau^{2}-3 \tau+1\right)}{\mu(1-\tau)^{3}} \\
q_{2} & = & \frac{3 \tau-1}{\mu^{2}(1-\tau)^{3}} \\
q_{3} & \frac{2}{\mu^{3}(1-\tau)^{3}}
\end{array}\right.
$$

Quando há a necessidade de extraplação, a matriz $\bar{Z}^{-1}$ é definida por $\Psi^{\prime}(z)=\operatorname{diag}\left(\frac{q_{3}}{2} z^{2}+q_{2} z+q_{1}\right)$.

O parâmetro de barreira $\mu$ é atualizado, se $\left\|\nabla L\left(\omega^{\mathbf{k}+\mathbf{1}}\right)\right\|_{\infty}>\varepsilon$, da seguinte maneira:

$$
\mu^{k+1}=0,618 \mu^{k}
$$

e faça-se o teste: se para todo $z$ obtermos que $z^{k+1} \geq-\tau \mu^{k+1}$, a heurística (1.24) permanece. Caso contrário, se para algum $z$ obtermos $z^{k+1}<-\tau \mu^{k+1}, \mu^{k+1}$ recebe o seguinte incremento:

$$
\mu^{k+1}=1,382 \mu^{k}
$$


e reinicie o procedimento previsor-corretor.

Com a estratégia de extrapolação cúbica (EX) definida e aplicada sobre o método MPIBLMCG, temos o método MPIBLMCG-EX.

\section{O MPIBLMCG-EX APLICADO AO PROBLEMA DE FLUXO DE POTÊNCIA ÓTIMO REATIVO.}

Aplicamos, através de uma implementação construída em MATLAB 6.1, o MPIBLMCGEX ao problema de fluxo de potência ótimo reativo (FPO-Reativo). O FPO-Reativo é um fenômeno observado e estudado no âmbito da engenharia elétrica em uma subárea denominada por sistemas de potências. Esse fenômeno é modelado matematicamente como um problema de otimização não-linear cuja região de viabilidade é não-convexa e o objetivo é o de minimizar as perdas de potência ativa na transmissão e que atenda aos seguintes critérios:

i) às leis de Kirchoff (restrições funcionais de igualdade);

ii) aos geradores que contém o controle reativo (restrições funcionais de desigualdade);

iii) às magnitudes dos fasores de tensão e ângulo em cada barra, em que a tensão é controlada por um limitante mínimo e máximo (variáveis canalizadas) e os ângulos são irrestritos.

O Modelo de um FPO-Reativo é definido, segundo [9], a seguir:

$$
\left\{\begin{aligned}
\text { Min } \quad f(\mathbf{x}) & =f(\mathbf{V}, \gamma)=\sum_{i=1}^{N L}\left(g_{k m}\right)_{i}\left(V_{k}^{2}+V_{m}^{2}-2 V_{k} V_{m} \cos \left(\gamma_{k}-\gamma_{m}\right)\right) \\
\text { s.a: } & \\
\Delta P_{t_{1}} & =P_{k}^{e s p}-V_{k}^{2} G_{k k}-V_{k} \sum_{m \in \Omega} V_{m}\left(G_{k m} \cos \left(\gamma_{k}-\gamma_{m}\right)+B_{k m} \operatorname{sen}\left(\gamma_{k}-\gamma_{m}\right)\right)=0 \\
\Delta Q_{t_{2}} & =Q_{k}^{e s p}+V_{k}^{2} B_{k k}-V_{k} \sum_{m \in \Omega} V_{m}\left(G_{k m} \operatorname{sen}\left(\gamma_{k}-\gamma_{m}\right)-B_{k m} \cos \left(\gamma_{k}-\gamma_{m}\right)\right)=0 \\
u_{1_{c r}} & \leq-V_{k}^{2} B_{k k}+V_{k} \sum_{m \in \Omega} V_{m}\left(G_{k m} \operatorname{sen}\left(\gamma_{k}-\gamma_{m}\right)-B_{k m} \cos \left(\gamma_{k}-\gamma_{m}\right)\right) \leq u_{2_{c r}} \\
l_{1_{b a r}} & \leq V_{b a r} \leq l_{2_{b a r}} \\
-\infty & \leq \gamma_{b a r} \leq \infty
\end{aligned}\right.
$$

em que: $f(\mathbf{x})$ : é a função objetivo, que representa as perdas de transmissão de potência ativa na transmissão na rede; $N L$ : é o número de linhas de transmissão que compõe o sistema; $\mathbf{V} \in \mathbb{R}^{N B}$ : é o vetor de tensões, tal que, $N B$ é o número de barras do sistema; $\gamma \in N B$ : Vetor de ângulos; $V_{k}$ : é a tensão na barra $k$ a conectar; $\gamma_{k}$ : é o ângulo da tensão da barra $k$ a conectar; $V_{m}$ : é a tensão na barra $m$ a ser conectada; $\gamma_{m}$ : é o ângulo da tensão da barra $m$ a ser conectada; $\left(g_{k m}\right)_{i}$ : é a condutância da linha $i$ a qual conecta as barras $k$ e $m ; \Delta P_{t_{1}}$ : é a equação de balanço 
de potência ativa. $\mathrm{O}$ sistema contém $(N B-1)$ equações; $P_{k}^{e s p}$ : é a diferença entre a Potência ativa gerada e a Potência ativa consumida na barra $k ; \Delta Q_{t_{2}}$ : é a equação de balanço de potência reativa. O sistema contém $N B C$ equações, onde $N B C$ é o número de barras de carga; $Q_{k}^{e s p}$ : é a diferença entre a Potência reativa gerada e a Potência reativa consumida na barra $k$; $c r$ : é o número de barras de controle de reativos $(N B C R)$; bar: é o número de barras do sistema; $\Omega$ : é o conjunto de todas as barras vizinhas à barra $k ; Y_{k m}=G_{k m}+j B_{k m}, j^{2}=-1$ : é a matriz de Admitância das linhas as quais $k$ conecta com $m \in \Omega$.

Neste trabalho, o ângulo da barra de referência, conhecida como barra slack, não será fixado em zero enquanto o MPIBLMCG-EX está em procedimento. Após o critério de convergência do MPIBLMCG-EX ser satisfeito, se definirmos por $\gamma_{\text {slack }}$ ao ângulo correspondente à barra slack, cada componente $\gamma_{j}, j=1, \ldots, n$, do vetor $\gamma$ é redefinido com a operação $\left[\left(\gamma_{j}-\gamma_{\text {slack }}\right) \frac{180}{\pi}\right]^{\circ}$, assim, o ângulo $\gamma_{j}$ está defasado em relação ao ângulo zero definido pela barra slack e a grandeza é apresentada em graus; os taps dos transformadores são todos fixos e, na existência de tap em uma linha de transmissão, utilizamos o inverso numérico desse valor para a construção da matriz $Y_{k m}$; utilizamos a potência de base 100 megawatts $(M W)$, isto é, 1 unidade (p.u.) equivale a $100 M W$.

\subsection{Resultados para o sistema IEEE-118.}

O banco de dados do sistema IEEE-118 barras pode ser encontrado em [10]. O sistema IEEE-118 barras contém 64 são barras de carga, 54 barras geradoras com controle de reativo e 186 linhas de transmissão. O sistema contém 181 restrições de igualdade, dentre as quais, 117 são do tipo $\Delta P$ e 54 são do tipo $\Delta Q$, e 54 restrições de desigualdades canalizadas; em que as canalizações das tensões e ângulos são definidas respectivamente por $\mathbf{0 , 9 5} \leq \mathbf{V} \leq \mathbf{1 , 1}$ $\mathrm{e}-\mathbf{9 9} \leq \boldsymbol{\gamma} \leq \mathbf{9 9}$.

Iniciamos o método com $\mathbf{V}^{\mathbf{0}}$ e $\boldsymbol{\gamma}^{\mathbf{0}}$ definidos pelo banco de dados; cada componente dos vetores dos estimadores dos multiplicadores de Lagrange $\boldsymbol{\delta}_{\mathbf{i}}^{\mathbf{0}} i=1, \ldots, 4$, é definida por $0,1 p . u$, $\mu_{0}=1, \beta_{0}=0,1, \tau=0,45$. Utilizamos (1.3) para obtermos $\mathbf{z}_{\mathbf{i}}^{\mathbf{0}}$ e $\lambda_{\mathbf{j}}^{\mathbf{0}}, i=1, \ldots, 4$, e $j=0, \ldots, 4$. A dimensão do vetor $\omega$ é 1577 , isto é, esse contém 1577 variáveis. A precisão utilizada é $\varepsilon=10^{-11}$ p.u. para a verificação das condições de KKT é o critério de parada do método.

A matriz $\hat{\theta}_{k}$ contém 55696 elementos, dentre os quais 3968 são não-nulos, isto é, a matriz contém $7,124 \%$ de elementos não-nulos. A matriz $\nabla \mathbf{g}\left(\mathbf{x}^{\mathbf{k}}\right) \hat{\boldsymbol{\theta}}_{k}^{-1} \nabla \mathbf{g}\left(\mathbf{x}^{\mathbf{k}}\right)^{\mathbf{T}}$ contém 32761 elementos, dentre os quais 32761 são elementos não-nulos. 
O método convergiu em 24 iterações. As perdas de potência ativa na transmissão são avaliadas em $f\left(\mathbf{x}^{*}\right)=107,7019055916945 M W$, a imagem pela função lagrangiana está avaliada em $L\left(\omega^{*}\right)=107,7019055917007 M W$, as avaliações nas restrições de igualdades são avaliadas em: $\max (\Delta P)=4,931166 \times 10^{-12}$ p.u. e $\max (\Delta Q)=6,661338 \times 10^{-13}$ p.u.; a avaliação do gap dual (condições de complementaridade) é avaliada em $\max ($ gap $)=5,900168 \times 10^{-15}$ p.u.; a precisão da solução ótima é avaliada em $\left\|\nabla L\left(\boldsymbol{\omega}^{*}\right)\right\|_{\infty}=4,931166 \times 10^{-12} p . u$; Os parâmetros $\mu \mathrm{e}$ $\beta$ são avaliados em $5,470932 \times 10^{-7}$ e 0,007161 , respectivamente. A tabela 1 apresenta os controles de reativos ótimos. Destacamos as barras 25, 34, 36, 62, 65, 66, 74, 85, 92 e 105, pois essas estão operando em seus limitantes inferiores ou superiores. Por serem restrições ativas em $\mathbf{x}^{*}$, seus respectivos multiplicadores de Lagrange são não-nulos. Esses estão avaliados, em p.u., respectivamente por: 0,$00407967 ; 0,001667840 ; 0,000174008 ; 0,000279097 ; 0,001130367$; 0,$009576484 ; 0,000068488 ; 0,002131085 ; 0,0036185407$ e 0,0006089243 .

Tabela 1: Os controles de reativos ótimos para o sistema IEEE-118 obtidos pelo método MPIBLMCG-EX.

AVALIAÇÃO DOS CONTROLES DE REATIVOS (em p.u.)

\begin{tabular}{c|c|c|c}
\hline Barra $k$ & $u_{1_{k}}$ & $\mathbf{h}\left(\mathbf{x}^{*}\right)$ & $u_{2_{k}}$ \\
\hline 1 & $-0,0500$ & 0,09452465944197 & 0,1500 \\
4 & $-3,0000$ & 0,56432228737156 & 3,0000 \\
6 & $-0,1300$ & 0,11756591896316 & 0,5000 \\
8 & $-3,0000$ & $-0,79294318711358$ & 3,0000 \\
10 & $-1,4700$ & $-1,02763757565558$ & 2,0000 \\
12 & $-0,3500$ & 0,32566134231543 & 1,2000 \\
15 & $-0,1000$ & $-0,05564030692245$ & 0,3000 \\
18 & $-0,1600$ & $-0,01315122236880$ & 0,5000 \\
19 & $-0,0800$ & 0,00027055038528 & 0,2400 \\
24 & $-3,0000$ & $-0,07266303239871$ & 3,0000 \\
25 & $-0,4700$ & $-0,47000000000000$ & 1,4000 \\
26 & $-10,0000$ & $-0,30749784815070$ & 10,0000 \\
27 & $-3,0000$ & 0,14191991299786 & 3,0000 \\
31 & $-3,0000$ & 0,00057491857410 & 3,0000 \\
32 & $-0,1400$ & $-0,00405381900876$ & 0,4200 \\
34 & $-0,0800$ & $-0,08000000000003$ & 0,2400 \\
36 & $-0,0800$ & $-0,08000000000001$ & 0,2400 \\
40 & $-3,0000$ & 0,08043343248677 & 3,0000 \\
42 & $-3,0000$ & 0,10729968406639 & 3,0000 \\
46 & $-1,0000$ & $-0,16604335982632$ & 1,0000 \\
49 & $-0,8500$ & $-0,10870287305296$ & 2,1000 \\
54 & $-3,0000$ & 0,03403698368626 & 3,0000 \\
55 & $-0,0800$ & $-0,01270144021316$ & 0,2300 \\
56 & $-0,0800$ & 0,00875291509913 & 0,1500 \\
\hline
\end{tabular}




\begin{tabular}{|c|c|c|c|}
\hline 59 & $-0,6000$ & $-0,06250394531306$ & 1,8000 \\
\hline 61 & $-1,0000$ & 0,24845942085262 & 3,0000 \\
\hline 62 & $-0,2000$ & $-0,20000000000001$ & 0,2000 \\
\hline 65 & $-0,6700$ & -0,66999999999999 & 2,0000 \\
\hline 66 & $-0,6700$ & -0,66999999999998 & 2,0000 \\
\hline 69 & $-99,0000$ & $-1,04059301668678$ & 99,0000 \\
\hline 70 & $-0,1000$ & $-0,04952869404970$ & 0,3200 \\
\hline 72 & $-1,0000$ & $-0,05730330608834$ & 1,0000 \\
\hline 73 & $-1,0000$ & $-0,02378392933632$ & 1,0000 \\
\hline 74 & $-0,0600$ & -0,05999999999999 & 0,0900 \\
\hline 76 & $-0,0800$ & 0,12228074736253 & 0,2300 \\
\hline 77 & $-0,2000$ & 0,28035141772669 & 0,7000 \\
\hline 80 & $-1,6500$ & $-0,39608764437143$ & 2,8000 \\
\hline 85 & $-0,0800$ & 0,23000000000000 & 0,2300 \\
\hline 87 & $-1,0000$ & 0,01166924045628 & 10,0000 \\
\hline 89 & $-2,1000$ & $-0,57420296361308$ & 3,0000 \\
\hline 90 & $-3,0000$ & 0,05434519123311 & 3,0000 \\
\hline 91 & $-1,0000$ & 0,02588264112604 & 1,0000 \\
\hline 92 & $-0,0300$ & 0,09000000000001 & 0,0900 \\
\hline 99 & $-1,0000$ & $-0,03619363335351$ & 1,0000 \\
\hline 100 & $-0,5000$ & 0,33209194465240 & 1,5500 \\
\hline 103 & $-0,1500$ & 0,01976219147427 & 0,4000 \\
\hline 104 & $-0,0800$ & $-0,00788005950646$ & 0,2300 \\
\hline 105 & $-0,0800$ & $-0,08000000000000$ & 0,2300 \\
\hline 107 & $-2,0000$ & $-0,08239741650467$ & 2,0000 \\
\hline 110 & $-0,0800$ & $-0,04574083181187$ & 0,2300 \\
\hline 111 & $-1,0000$ & $-0,00338931411819$ & 10,0000 \\
\hline 112 & $-1,0000$ & $-0,00851631749361$ & 10,0000 \\
\hline 113 & $-1,0000$ & $-0,07893380174018$ & 2,0000 \\
\hline 116 & $-10,0000$ & $-0,34608185144089$ & 10,0000 \\
\hline
\end{tabular}

A figura 1 apresenta a sequência de soluções das tensões obtidas pelo método ao longo das iterações. 


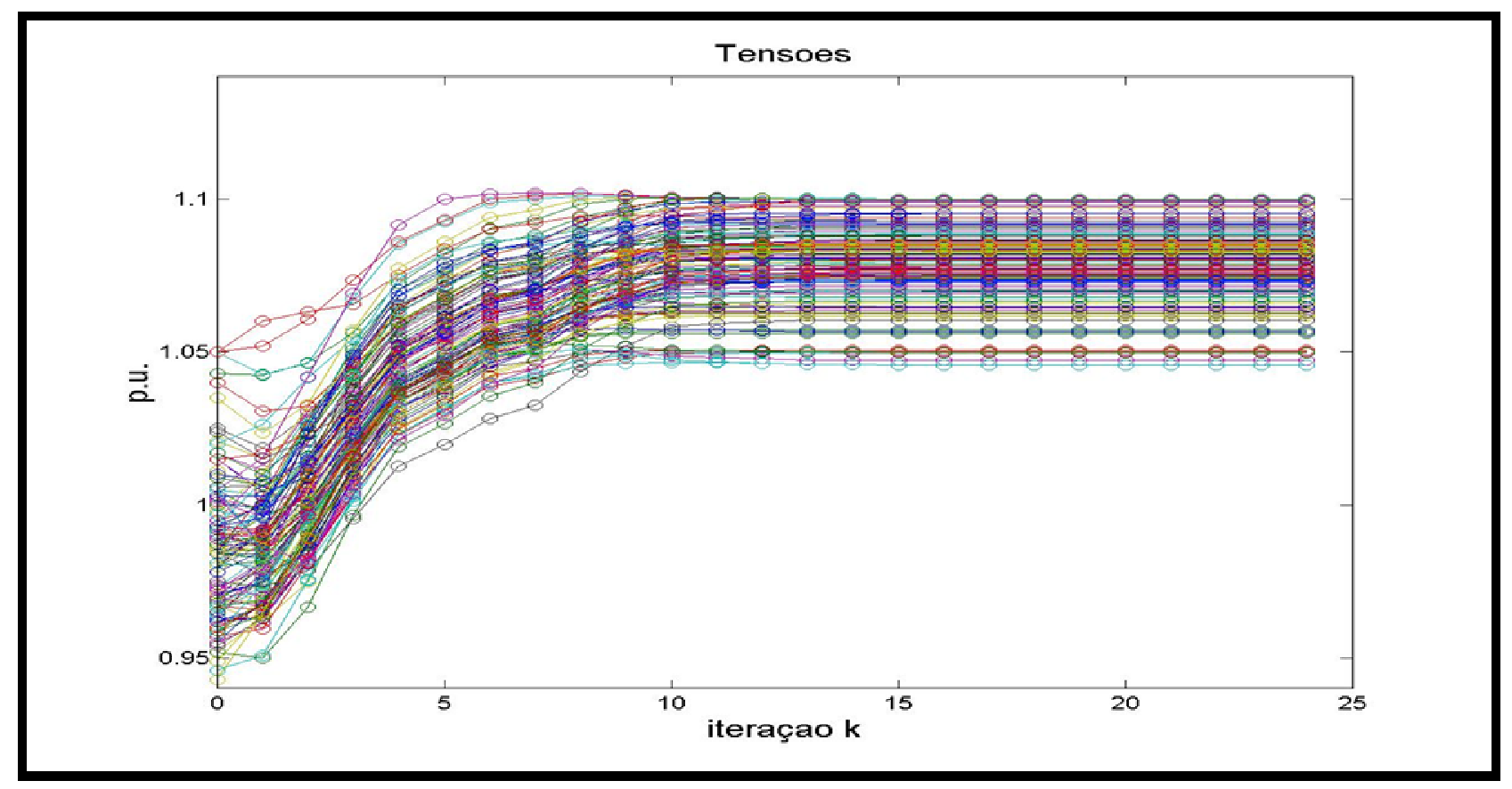

Figura 1: Sequência dos pontos $\mathbf{V}^{\mathbf{k}}$ obtidos pelo método MPIBLMCG-EX para o sistema IEEE118.

Notamos na figura 2 que as tensões $V_{4}, V_{9}, V_{25}, V_{37}, V_{66}, V_{69}, V_{80}, V_{87}, V_{89}$ e $V_{100}$ estão operando em seus limites máximos. Por serem restrições ativas em $\mathbf{V}^{*}$, seus respectivos multiplicadores de Lagrange são não-nulos. Esses estão avaliados, em p.u., respectivamente por: 0,$0320225 ; 0,1877409 ; 0,3438780 ; 0,0730942 ; 0,6053443 ; 0,2065799 ; 0,0592597 ; 0,0055223$; 0,4864108 e 0,0546143 . 


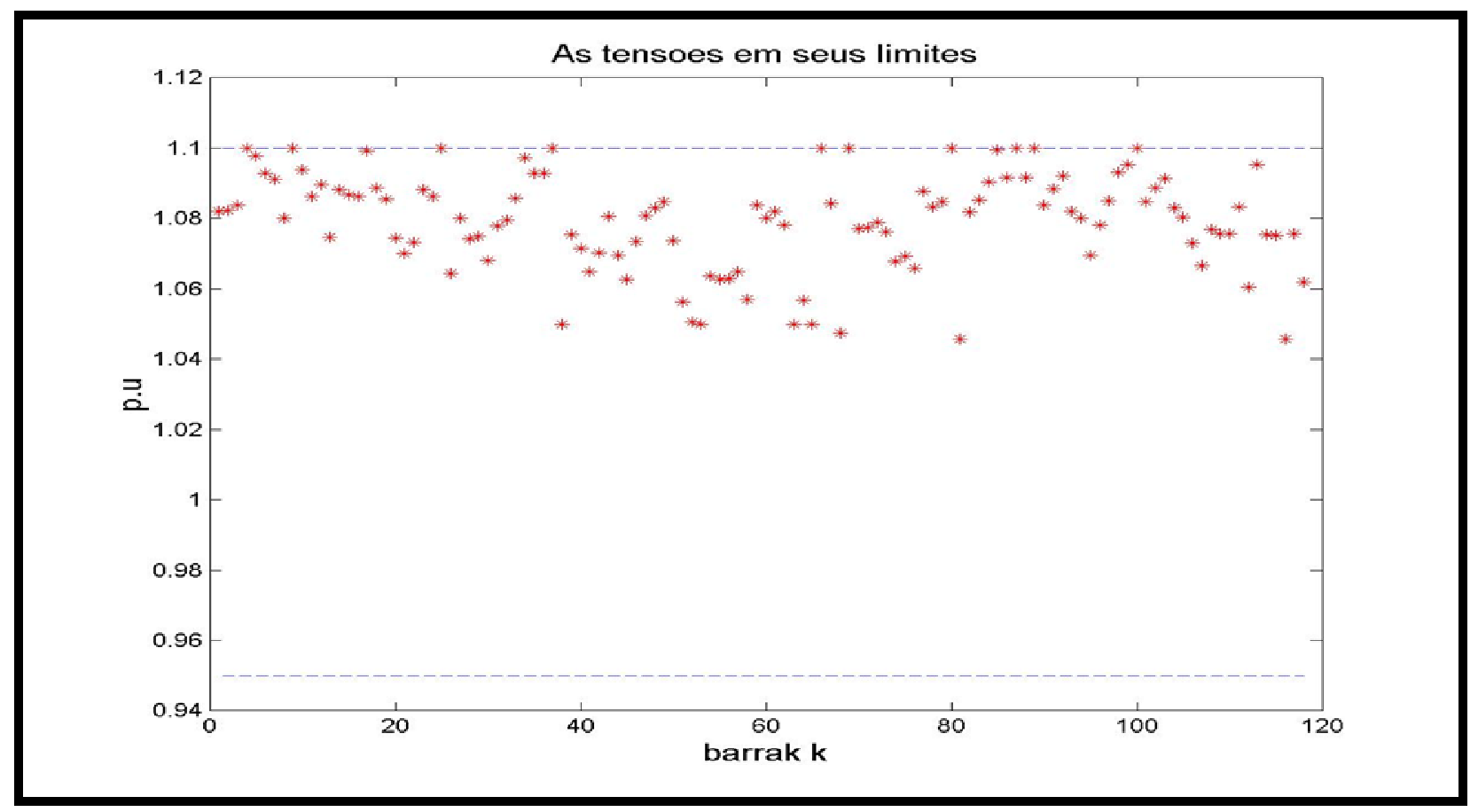

Figura 2: As tensões ótimas determinadas pelo MPIBLMCG-EX em seus limites de definição para o sistema IEEE-118.

A figura 3 apresenta $\gamma^{*}$, em graus:

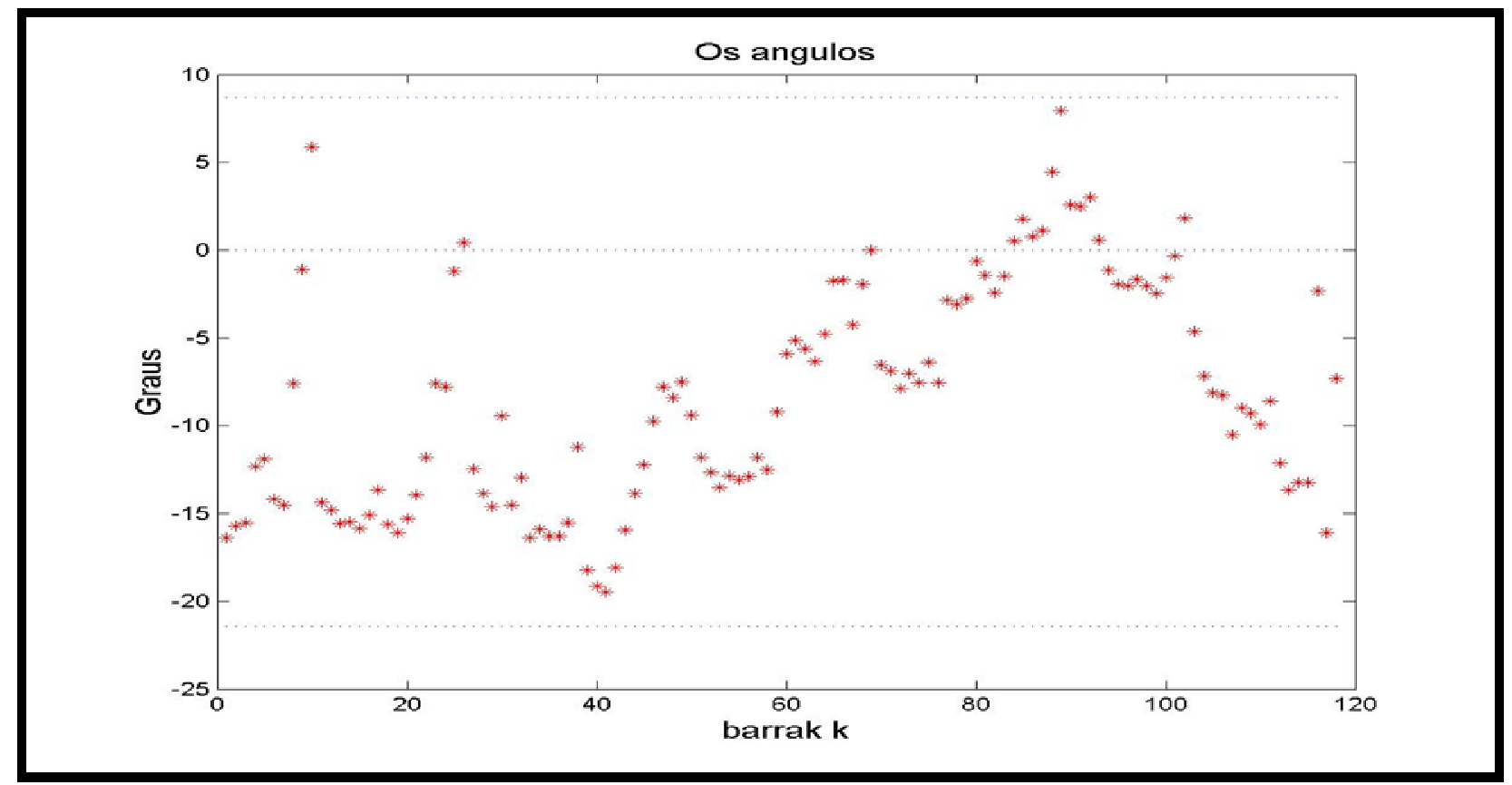

Figura 3: Os ângulos ótimos determinados pelo MPIBLMCG-EX para o sistema IEEE-118. 
A figura 4 apresenta a sequência de multiplicadores de Lagrange $\lambda_{\mathbf{0}}^{\mathbf{k}}$ gerados pelo método:

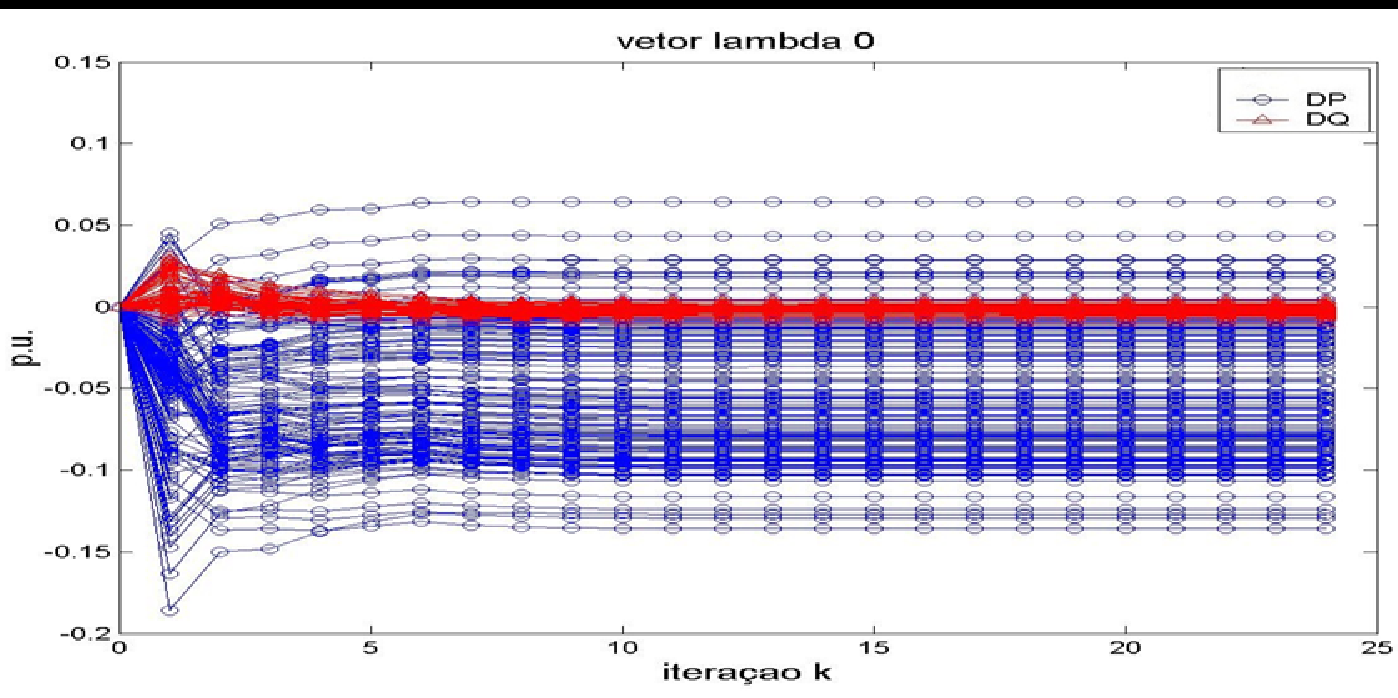

Figura 4: Sequências de pontos $\lambda_{\mathbf{0}}^{\mathbf{k}}$ gerados pelo MPIBLMCG-EX para o sistema IEEE-118.

A figura 5 apresenta a sequência de multiplicadores de Lagrange $\lambda_{\mathbf{i}}^{\mathbf{k}}, i=1, \ldots, 4$, geradas pelo método.

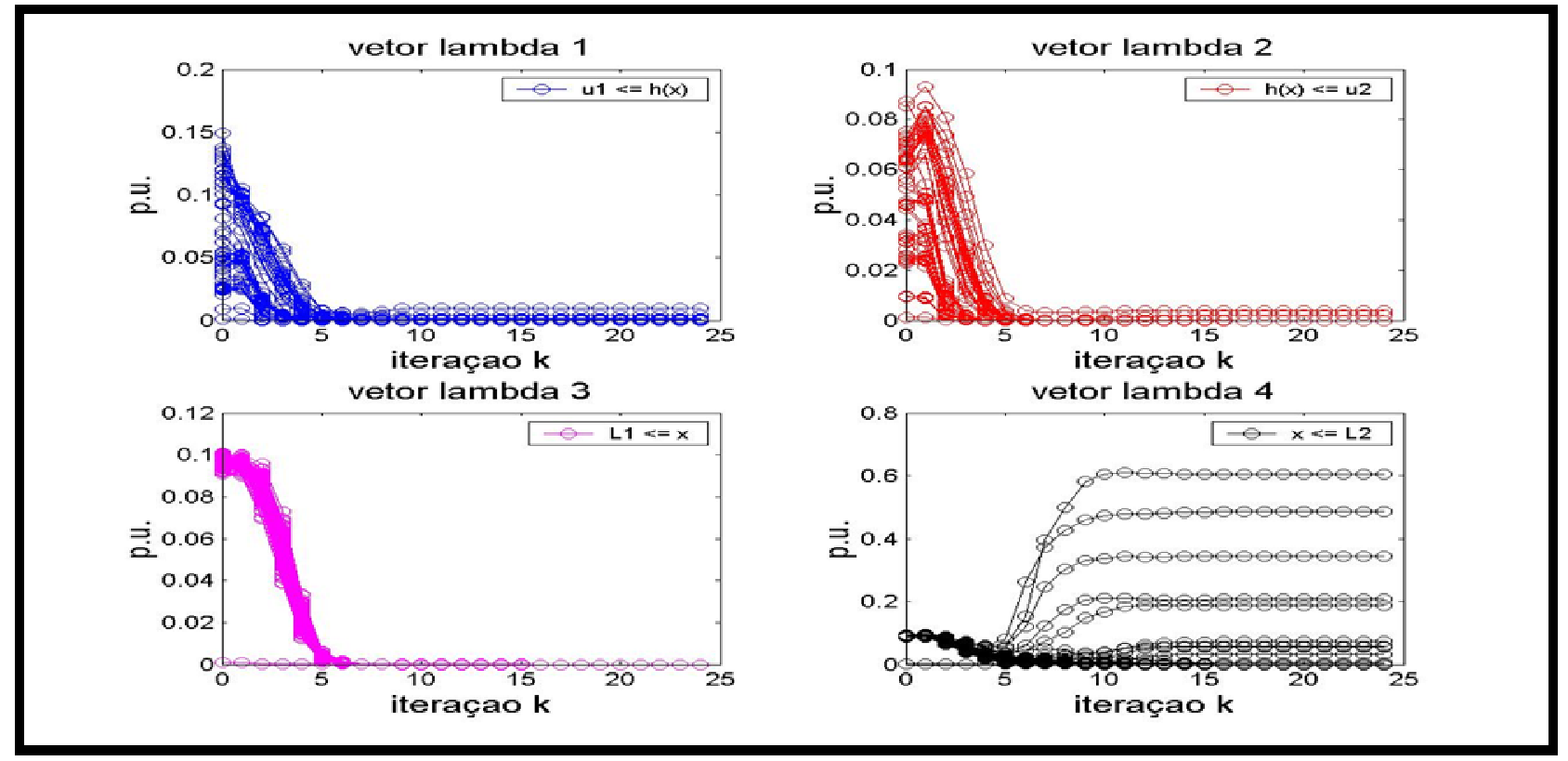

Figura 5: Sequências de pontos $\lambda_{\mathbf{i}}^{\mathbf{k}}, i=1, \ldots, 4$, geradas pelo método MPIBLMCG-EX para o sistema IEEE-118. 
A figura 6 apresenta a sequência de valores da função objetivo obtida pelo método em cada iteração.

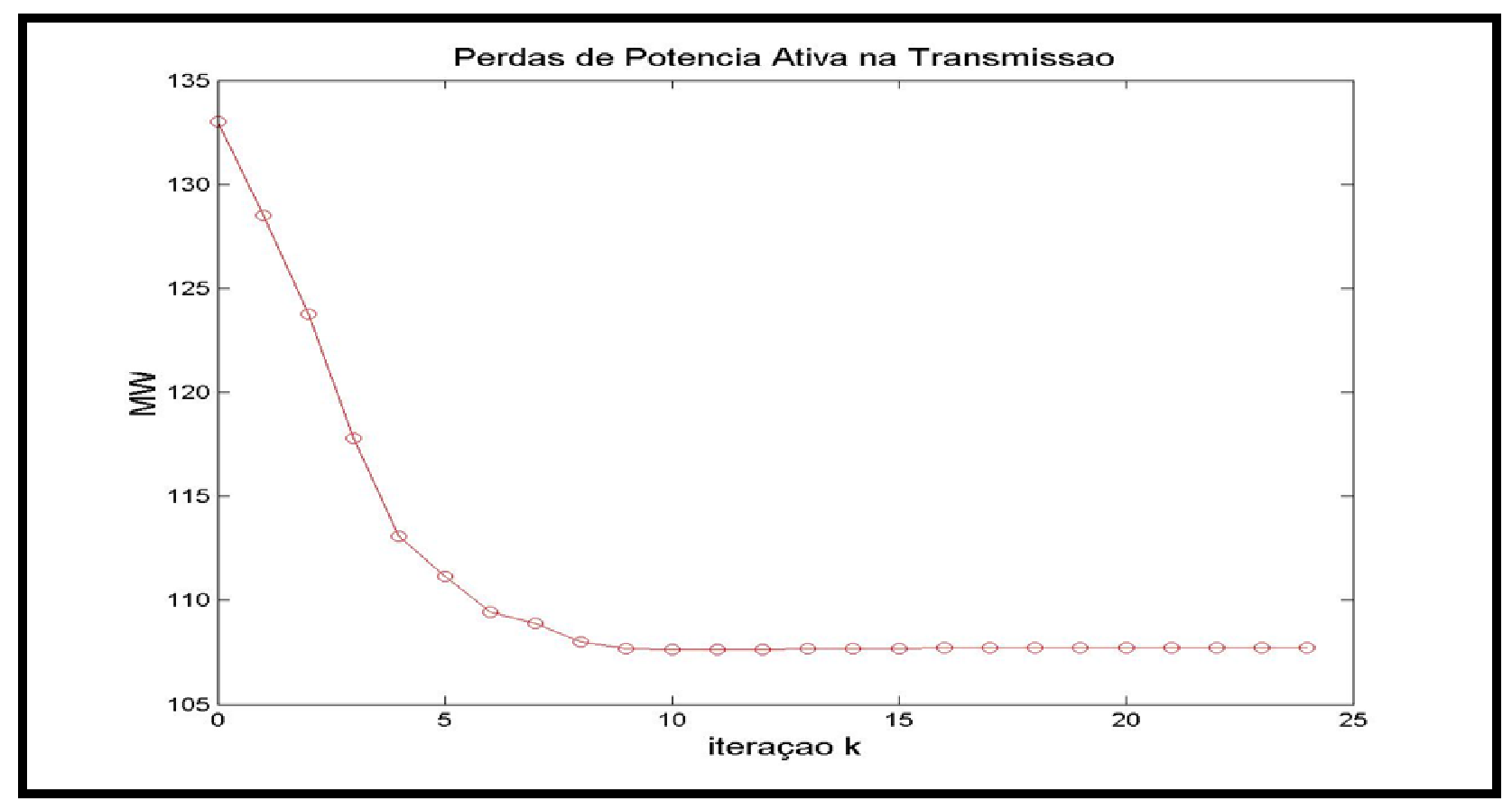

Figura 6: Sequência de valores da função objetivo gerados pelo MPIBLMCG-EX para o sistema IEEE-118.

A figura 7 apresenta a evolução do parâmetro de amortecimento $\beta_{k}$ ao longo das iterações. 


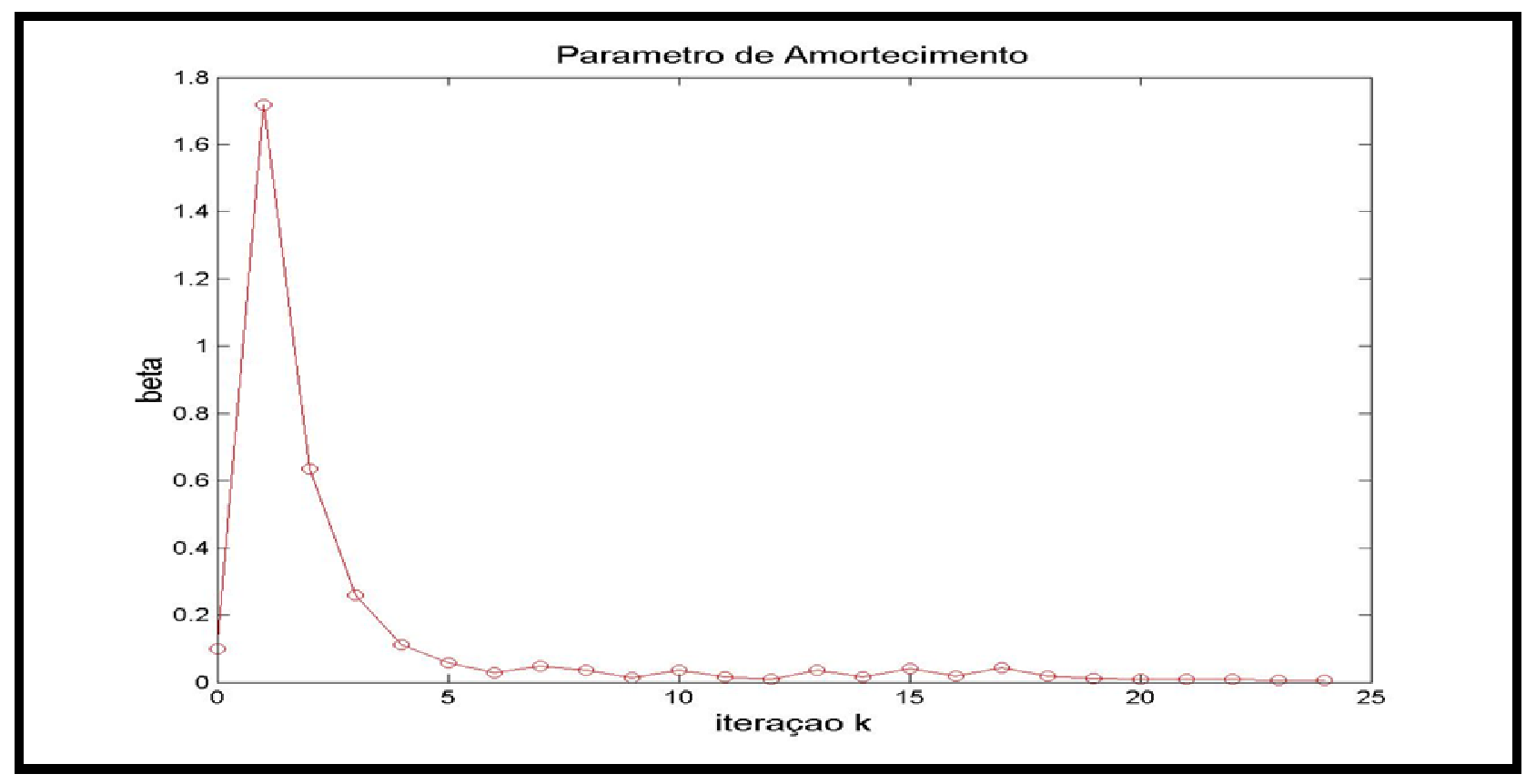

Figura 7: Evolução do parâmetro de amortecimento $\beta_{k}$ gerado pelo MPIBLMCG-EX para o sistema IEEE-118.

Na figura 7 observamos que a $\theta_{k}$ necessitou de um salto no amortecimento para torná-la definida positiva, na $1^{\mathrm{a}}$ iteração. O método, a partir dessa iteração, utilizando-se dos critérios apresentados em (1.19) a (1.21), gerou uma sequência decrescente para o parâmetro de amortecimento até a $5^{\mathrm{a}}$ iteração. Esta sequência apresenta oscilações de baixa amplitude entre as iterações 6 a 18 até se estabilizar, em um valor finito em torno de 0,007161, a partir da 19a iteração. Esse valor nos mostra quão mal condicionada ou quão instável é a matriz $\theta_{k}$ para uma sutil mudança de direção de busca quando $\mathbf{x}^{\mathbf{k}} \rightarrow \mathbf{x}^{*}$, pois $\beta^{k}$ não tende para zero, isto é, algum autovalor da matriz hessiana torna-se negativo e a Decomposição de Cholesky não é possível. $\mathrm{O}$ autovalor que causa essa instabilidade pertence à linha e coluna 120 da matriz $\mathbf{D}$ de autovalores de $\theta_{k}$ o qual é avaliado, segundo o comando "eig $\left(\theta_{k}\right)$ " do MATLAB, em -0,094588. Após a aplicação da estratégia global, apresentada na seção 2, esse autovalor é avaliado em 17,163110 e, assim, o MPIBLMCG-EX tem condições suficientes de gerar uma nova direção de descida.

\section{CONCLUSÕES.}

Neste trabalho apresentamos um método previsor-corretor primal dual de pontos interiores e exteriores barreira logarítmica modificada, com estratégias de extrapolação cúbica e de convergência global (MPIBLMCG-EX). O método foi eficiente devido aos seguintes aspectos:

i) ao procedimento previsor-corretor, por considerar todos os resíduos condizentes às viabilidades primal, dual e condição de complementaridade. Portanto, esse contribuiu ao 
refinamento das direções de busca, ao tratamento da esparsidade da matriz dos coeficientes $A_{k}$ do sistema (1.5) e também por explicitar as equações de cada uma das direção de busca;

ii) ao procedimento barreira logarítmica modificada, pois esse possibilita que convergência do método para pontos pertencentes à fronteira da região viável do problema (1.1) de forma exata e não-assisntótica, enquanto que, no procedimento barreira logarítmica clássica de [11], a convergência, se ocorrer, é assintótica só possibilita a obtenção de pontos da fronteira de forma aproximada.

iii) à estratégia de convergência global, a qual é uma variante do método de LevenbergMarquardt que ajusta os autovalores diagonais da matriz hessiana $\theta_{k}$, tornando-os positivos de tal forma que $\theta_{k}$ torna-se diagonalmente dominante e é transformada em definida positiva. Essa estratégia é interessante, pois não utilizamos procedimento de busca linear para o cálculo do passo, mesmo que a região de viabilidade é não-convexa e por garantir que a matriz hessiana é não-singular a medida que $\boldsymbol{\omega}^{\mathbf{k}} \rightarrow \boldsymbol{\omega}^{*}$, isto é, a estratégia também evitou a instabilidade numérica, se no critério de parada considerarmos $\varepsilon>0$ muito pequeno;

iv) à estratégia de extrapolação cúbica ou de ajuste cúbico, a qual auxiliou ao MPIBLMCG em ocasiões extremas em que uma variável de folga viesse a transpor a região de viabilidade relaxada ou não atender o critério de que $z \geq-\tau \mu$. Esta extrapolação é definida através de um polinômio que preserva as diferenciais de primeira e de segunda ordem do logaritmo no ponto $z=-\tau \mu$. Essa estratégia nos permitiu a aplicação de uma nova heurística para a atualização do parâmetro de barreira em problemas de programação não-linear e nãoconvexo, de tal forma a incrementar esse parâmetro na iterações em que o ajuste cúbico fosse necessário e reduzi-lo a uma taxa $\alpha_{\mu}$ superior a 0,5 . Observamos que, em problemas de FPOReativo, para $\alpha_{\mu}>0,15$ a possibilidade de que o logaritmo modificado não esteja definido para algum $z<-\mu$, numa iteração $k$.

Considerando-se aos aspectos apresentados acima, o MPIBLMCG-EX, o qual foi aplicado ao problema de FPO-Reativo, que é um problema de otimização não-linear e nãoconvexo, sobre o sistema elétrico de potência de 118 barras, mostrou-se robusto por resolvê-lo e eficiente pelo fato de que foram necessárias 24 iterações para obtermos a solução ótima do problema de maneira que o critério de parada fosse satisfeito em relação à precisão $\left\|\nabla L\left(\boldsymbol{\omega}^{24}\right)\right\|_{\infty} \leq 10^{-11}$. 


\section{REFERÊNCIAS.}

[1] Sousa V. A., "Resolução do Problema de Fluxo de Potência Ótimo Reativo Via Método da Função Lagrangiana Barreira Modificada". Tese (Doutorado), São Carlos: Escola de Engenharia de São Carlos, Universidade de São Paulo, 2006.

[2] Polyak, R.,"Modified barrier functions." Mathematical Programming, vol. v.54, n. 2, p. 177 $-222,1992$.

[3] Wu, Y., Debs, A. S., Marsten. R. E., “A Direct Nonlinear Predictor- Corrector Primal-Dual Interior Point Algorithm for Optimal Power Flow," IEEE Transactions on Power Systems, vol. 9, pp. 876-883, 1994.

[4] Granville, S., "Optimal Reactive Dispatch Through Interior Point Methods," IEEE Transactions on Power Systems, vol. 9, pp. 136-146, 1994.

[5] Bazaraa, M. S., Sherali, H. D., Shetty, C. M., Nonlinear Progremming: Theory and Algorithms, 3 ed., New Jersey: Wiley Interscience, 2006.

[6] Benson, H. Y., Shanno, D. F., Vanderbei, R. J., "Iinterior-Ponit Methods for noncovex nonlinear programming: Jamming and comparative numerical testeing," Operations Research and Financial Engineering, 2000.

[7] Pereira, A. A., "O método da função lagrangiana barreira modificada/penalidade." Dissertação (mestrado), São Carlos: Escola de Engenharia de São Carlos, Universidade de São Paulo, 2007.

[8] Matioli, L., "Uma nova metodologia para construção de funções de penalização para algoritmos de lagrangeano aumentado".Tese (Doutorado), Florianópolis, Santa Catarina: Programa de Pós-Graduação em Engenharia de Produção, Universidade Federal de Santa Catarina, 2001.

[9] Monticelli, A., "Fluxo de carga em redes de energia elétrica", São Paulo: Edgard Blücher , 1983.

[10] University Washington Electrical Engineering. [Online]. Available: http://www.ee.washington.edu/research/pstca/. [Acesso em 26 Março 2012].

[11] Frisch, K. R., “The Logarithmic Potential Method for Convex Programming,” 1955. 